\title{
Functional Roles and Biological Mechanisms of Circular RNAs and Their Encoded Peptides in Glioma: A Narrative Review
}

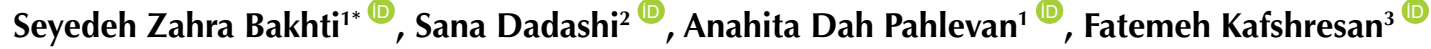 \\ ${ }^{1}$ Department of Biology, Faculty of Sciences, University of Mohaghegh Ardabili, Ardabil, Iran \\ ${ }^{2}$ Department of Microbiology, Sarab Branch, Islamic Azad University, Sarab, Iran \\ ${ }^{3}$ Department of Cell Sciences Research Center (Isfahan Campus), Royan Institute, ACECR, Tehran, Iran
}

\begin{abstract}
Circular RNAs (circRNAs) are a complicated class of non-coding RNAs that have a covalently closed loop structure and are very stable and cautious. Multiple biological processes of malignancy, including tumorigenesis, development, invasion, metastasis, apoptosis, and vascularization, are disrupted by an increased number of circRNAs. Recent research has showed that circRNAs, functioning as microRNA (miRNA) sponges or protein scaffolds, interacting with RNA-binding proteins (RBPs), and autophagy regulators, affect the transcription and splicing regulation. Many circRNAs have tissue-specific expression patterns and are heavily conserved. CircRNA levels in neurons are dynamically modulated. Growing evidence suggests that circRNAs are highly abundant in neural tissues, perhaps owing to the proliferation of particular genes that promote circularization, implying that circRNA dysregulation is linked to nervous system disorders including glioma. The most widespread and deadly primary malignant brain tumor is glioma. CircRNA has a close connection to glioma, according to reported research. Here, the current knowledge about the properties of circRNAs is introduced and the biological and molecular functions of circRNAs are described. Then, the clinical association of circRNAs with glioma/glioblastoma and their level of expression and their regulatory mechanisms in tumorigenesis are discussed. Moreover, the potential of circRNAs as diagnostic biomarkers and predictors of brain cancer risk and possible therapeutic targets in medicine is examined.
\end{abstract}

Keywords: CircRNAs, miRNA, Brain, Glioma, Glioblastoma
*Correspondence to Seyedeh Zahra Bakhti, Ph.D. of Cell and Molecular Biology, Department of Biology, Faculty of Sciences, University of Mohaghegh Ardabili, Ardabil, 56199-11367 Iran. Telefax: +98-45-33514701,

Email: s.z.Bakhti@gmail.com

Published online 30 October 2021

Citation: Bakhti SZ, Dadashi S, Dah Pahlevan A, Kafshresan F. Functional roles and biological mechanisms of circular rnas and their encoded peptides in glioma: a narrative review. Clin Neurosci J. 2021;8(4):157-167. doi:10.34172/icnj.2021.33.

\section{Introduction}

Glioma is a prevalent central nervous system (CNS) tumor, involving $80 \%$ of all malignant brain tumors and $30 \%$ of all CNS tumors, ${ }^{1}$ and it comes in a variety of malignancy grades and histological subtypes. ${ }^{2}$ Glioma is the prevalent cause of death from main brain tumors, and it is also a particularly deadly cancer worldwide. Glioblastoma multiforme (GBM), the highly widespread and aggressive glioma, has a median total survival rate of about 15 months that has stayed constant for decades. ${ }^{3}$ Gliomas are primary brain tumors believed to be caused by neuroglial stem or progenitor cells. Rapid cell proliferation and angiogenesis are hallmarks of gliomas. ${ }^{4}$

Non-coding RNAs (ncRNAs) have been shown to affect biological processes that control glioma progression and initiation in a number of studies. ${ }^{5}$ Circular RNAs (circRNAs) are circular single-stranded RNA molecules that do not have $5^{\prime}$ caps and a $3^{\prime}$ poly A tails. CircRNAs are formed by back-splicing from precursor mRNA
(pre-mRNA), where an upstream acceptor site is linked to a donor site. ${ }^{6}$ CircRNAs are resistant to exonucleases, including RNase $\mathrm{R}$, and have a longer half-life than linear mRNAs. ${ }^{7-9}$ CircRNAs have a stable and conserved closedloop structure and have different degrees of expression in different organs and tissues of the body and play an important role in the onset and progression of diseases such as cancer through different pathways. ${ }^{10}$

CircRNA has a covalent closed-loop structure and is an extremely stable and conservative special RNA that is commonly distributed in different tissues and organs with varying expression degrees and plays a role in the prevalence and progression of diseases such as cancer in a variety of ways. ${ }^{10}$ A large study of more than 2000 different cancer samples showed that circRNAs affect the pathogenesis of many cancers. ${ }^{11}$ CircRNAs are often involved in various oncogenic processes ${ }^{11-13}$ because of their dysregulation in multiple cancers. CircRNAs affect tumor development, metastasis therapy resistance, and 
EMT transformation. ${ }^{14}$ Rybak-Wolf et al $^{15}$ discovered that circRNAs are plentiful and unique in neural and brain tissues, implying that they affect neural separation and neurological disorders. It has also been reported that circRNAs can cross the blood-brain barrier through exosomes and are present in cerebrospinal fluid (CSF). ${ }^{16}$ CSF, produced by choroid plexuses, ${ }^{17}$ is in direct contact with the extracellular fluid of the brain and has a variety of functions, including mechanical and chemical protection of the brain, nutrient transfer and biological substances, and removal of toxic metabolites. ${ }^{18-20}$ Therefore, the study of the presence of circRNAs in CSF can be considered as an important ideal biomarker for the diagnosis and control of some types of brain tumors. ${ }^{16}$ Multiple recent experiments have revealed that circRNAs are highly abundant in neural tissues than other tissues, and that they have differential expression patterns in various brain regions. This may be due to the proliferation of proteincoding genes that produce numerous circRNAs, as well as splicing factors and RNA-binding proteins (RBPs) that regulate circRNA formation. ${ }^{21}$ Brain-specific genes can acquire further sequence properties that foster RNA circularization, and broad cis-element and trans-factor regulation may lead to a higher abundance of circRNAs in the brain. ${ }^{21,22}$ CircRNAs in glioma specimens, on the other hand, are lower than in normal samples, ${ }^{12}$ this suggests that circRNAs may have different roles and functions in the development of glioma.

Understanding the role and circRNAs mechanism in glioma/GMB tumorigenesis could lead to the creation of new tracing procedures and useful treatment steps. In this study, current advances in the biological functions and molecular mechanisms of circRNAs and the peptides encoded by them in glioma/GMB tumorigenesis were reviewed. In the following, the clinical association of circRNAs with glioma/GMB and their expression level and regulatory mechanisms and their role as oncogenes or suppressors of tumor in tumorigenesis are discussed. Moreover, the potential of circRNAs as diagnostic biomarkers and predictors of brain cancer risk and possible therapeutic targets in medicine are explored.

\section{Functions of cirRNAs}

CircRNAs with enzyme and substrate binding sites can serve as protein scaffolding to mediate protein-protein interactions. CircRNAs are also involved in transcription, translation and alternative splicing of genes, interaction with RBP, and cell autophagy; hence, they affect the biological function of cancer $^{23-25}$ (Figure 1).

\section{CircRNAs Act as microRNA Sponges in Glioma}

Since many circRNAs have miRNA binding sites and miRNA interaction elements, functioning as a "miRNA sponge" is the notable significant role and process for regulation of human cancer progression and growth, especially brain tumors (Figure 2). They affect tumorigenesis due to their ability to bind proteins. ${ }^{26}$ Parts of circRNAs with miRNA binding sites have been shown to behave as sponges, thus binding and interacting with miRNAs and regulating their functions, and influencing the expression and activation of target genes, which are located downstream of them. ${ }^{27,28}$ According to Zheng's research, circ-TTBK2 stimulated glioma proliferation and prevented apoptosis by serving as a sequencespecific miR-217 sponge. ${ }^{29}$ Different miRNAs, like miR124-3p and miR-654, may be sponged by circ-HIPK3. ${ }^{30}$ Circ-HIPK3 facilitated glioma development via the circ-HIPK3/miR-654/IGF2BP3 network. ${ }^{31}$ MiR-654 was established as a circ-HIPK3 target, and miR-654 targeted IGF2BP3. Circ-PTN improved Nestin, SOX9, CD133, and SOX2 expression by sponging miR-330-5p and miR145-5p, promoting glioma development and stemness. ${ }^{32}$ Circ_0034642 and circ_0076248 have previously been shown to facilitate glioma invasion and proliferation in the miR-181a/SIRT1 and miR-1205/BATF3 axis, respectively. ${ }^{33,34}$ CircRNAs could also regulate GMB initiation and progression by acting like a "miRNA sponge." CircMMP9, through sponging miR-124 in GBM cells, may regulate the eukaryotic initiation factor $4 \mathrm{~A} 3$ (eIF4A3) expression, speed up migration, invasion, and proliferation. ${ }^{35}$ Circ_0029426, through sponging miR197, promotes cell proliferation and growth, invasion and migration while also inhibiting GBM cell apoptosis. ${ }^{36}$ According to a recent analysis, circSMARCA5 serves as a sponge for the splicing factor serine and arginine rich splicing factor 1 (SRSF1), responsible for VEGFA expression in $\mathrm{GBM}^{37}$ CircNT5E acts as a miR-422a sponge and influences GBM cell apoptosis, invasion, and migration capacity by up-regulating the levels of NT5E,

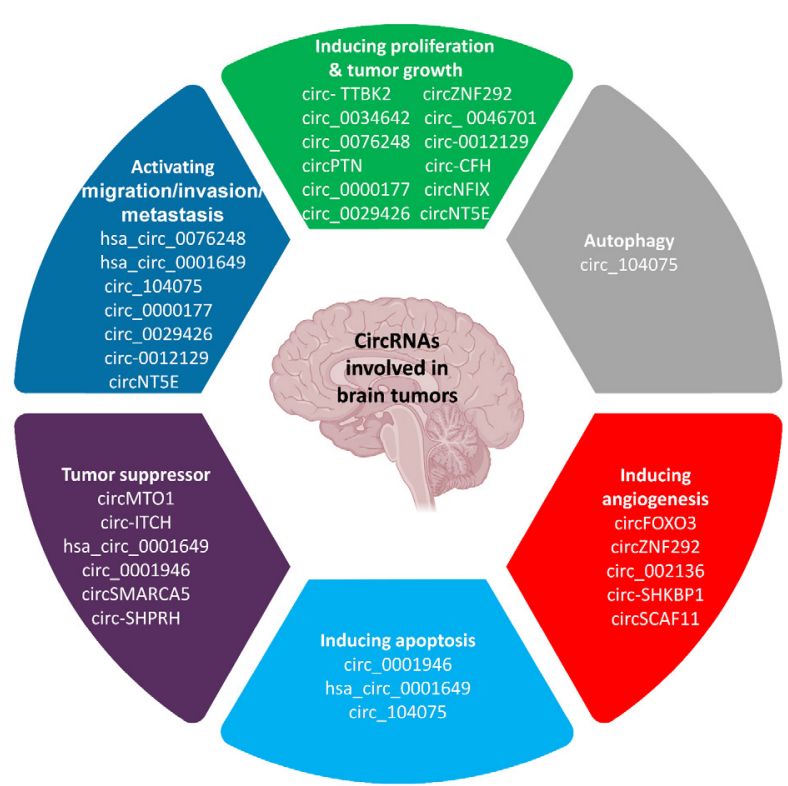

Figure 1. Mechanisms and Biological Functions of circRNAs in Brain Tumors. 


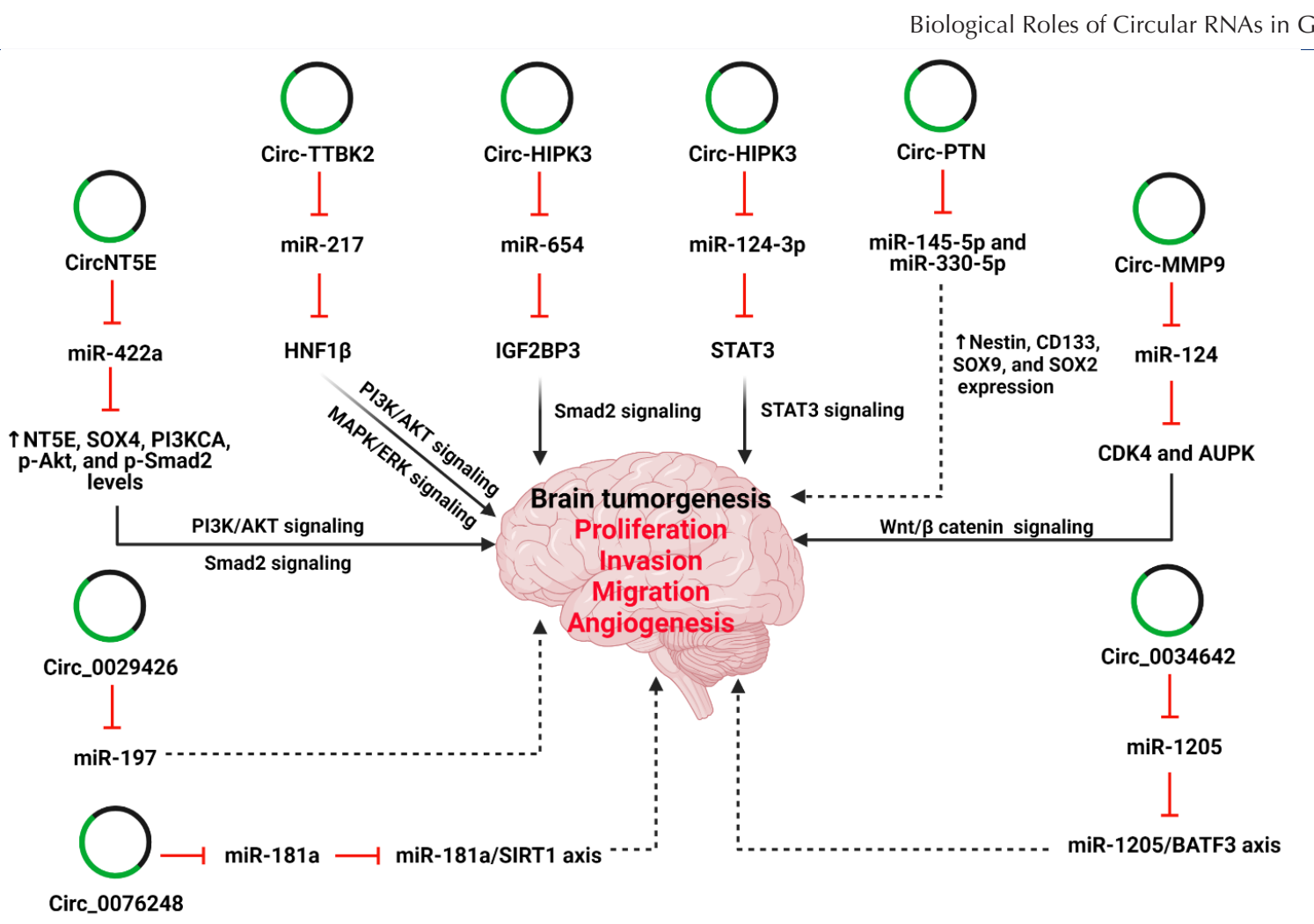

Figure 2. The present diagram shows the regulatory relationship of circRNA-miRNA-mRNA networks with different signaling pathways that are involved in brain tumorigenesis. Several circRNAs have been shown to act as miRNA sponges, thereby regulating the expression level of downstream genes. These target genes or proteins, by acting as regulatory proteins or transcription factors and other mechanisms, regulate factors associated with tumor signaling pathways.

p-Smad2, PI3KCA, p-Akt and SOX4..$^{38}$ circRNAMMP9, which also spongs miR-124, was found to be up-regulated in GBM by Wang et al. ${ }^{35}$ CiRS-7 (circRNA sponge for miR7), as the best known circRNA, was found to be heavily expressed in the brain and to have over 70 conserved target sites for miR-7.39 CiRS7 overexpression tightly bound $\mathrm{miR}-7$, facilitating the unique interaction of miR7/AGO2, reducing the CiRS-7 ability to bind and interact with target mRNAs. These results appeared to confirm that circRNAs acting as miRNA sponges is a prevalent occurrence. However, it's been debated whether miR7 and miR-671 shape a regulatory network in the brain through sponge function, and whether the ciRS-7 and miR-671 combination causes AGO2-mediated cleavage of ciRS-7 rather than miR-7 inhibition. ${ }^{40,41}$ Furthermore, many circRNAs had less miRNA binding sites, implying that several circRNAs could not act as miRNA sponges. As a result, further research is needed to determine the impact of circRNAs as miRNA inhibitors or scaffolds.

\section{CircRNAs Act as Autophagy Regulator in Glioma}

Autophagy, as a conserved and sequential process of self-destruction, occurs in response to cellular stress and survival, and is often involved in tumor progression, progression, and metastasis. ${ }^{42,43}$ CircRNAs have been shown to play an important role in tumor autophagy, and affect the occurrence and growth of human cancer, according to new research. Furthermore, circ 104075 was linked to glioma cell autophagy and apoptosis. In glioma U251 cells, circ 104075 overexpression neutralized Matrine's inhibitory impact on proliferation and enhanced cell autophagy. ${ }^{44}$ Nonetheless, research on the importance of circRNAs in tumor autophagy is in its early stages, and the real role and function, as well as the possible mechanisms underlying this, must be investigated in the future.

\section{CircRNAs Interact With RBPs and Act as a Protein Scaffold}

CircRNAs may bind to specific proteins to make circRNPs (circRNA protein complexes), which then regulate the function of related proteins, protein subcellular localization, and the transcription of parental or related genes. CircRNAs are involved in multiple human diseases through various mechanisms. ${ }^{45}$ By functioning as a sponge, they may decrease the inhibitory impact of miRNAs on messenger RNA (mRNA) ${ }^{46,47}$ Furthermore, by serving as dynamic scaffolds, circRNAs interacts with RBPs and regulate their function. ${ }^{6,48}$ CicRNAs work as protein sponges, miRNA sponges, or scaffolds, and splicing activity regulators. ${ }^{23}$ CircRNA binds to RBPs and impairs their role. RBPs are a family of proteins that play a critical role in gene translation and transcription. ${ }^{49}$ RBPs could be sequestered, stored, and sorted by circRNA, 
allowing it to regulate their intracellular localization. ${ }^{50,51}$ RBP quaking has been shown to modulate the circRNA shape by creating RNA-protein complexes (RPCs) in recent studies. ${ }^{52}$ Furthermore, RBM20 (RNA-binding motif protein 20) affected the development of a subset of circRNAs, forming the RBM20-dependent circRNAs class. ${ }^{53}$ As a result, circRNA and RPBs are strongly linked to the prevalence and progression of cancer. Overall, circRNAs influence cell migration, proliferation, growth, invasion, and other pathological processes and are expressed abnormally and linked to the human cancer emergence and progression. CircRNA also interacts with protein compared with RBPs. It may serve as protein sponges by adsorption of one or more proteins through their binding sites, mediating protein interaction directly by functioning as protein scaffolding, and thereby regulating the expression of gene. In tumors, CDK2 (cyclin-dependent kinase 2) and p21 proteins, for example, are correlated to cell cycle control. CircFOXO3 can act as a scaffold for the development of the circFOXO3p21-CDK2 ternary complex, inhibiting CDK2 role and thereby influencing cancer cell cycle progression. ${ }^{54}$

CircRNAs Play a Role in Gene Alternative Splicing, Translation, and Transcription Processes

The circRNA in the nucleus served as splicing or transcriptional controls, interfering with expression of gene and participating in alternative transcription and splicing. For instance, in tumor cells, circPOK that is originated from the $z b t b 7 a$ gene affected the transcription of gene via encoding the Pokemon transcription factor, hence regulation of the pro-angiogenic and proproliferative factors by activation of ILF2/3 (interleukin enhancer-binding factor 2 and 3) complex..$^{55}$ Furthermore, by inhibiting RREB1 (RAS-responsive element-binding protein 1) transcription factor through the Ras pathway, circITGA7 increased the transcription of its host itga7 (integrin Subunit alpha 7) gene. ${ }^{56}$ Gene transcription, alternative splicing, and translation are critical processes to exert biological functions in cancers, but circRNA highly influences these processes.

CircRNAs Can Be Translated Into Peptides That Are Related to Glioma

They have ORFs in their sequences, according to recent research. ${ }^{57-59} \quad \mathrm{~N}^{6}$-methyladenosine $\quad\left(\mathrm{m}^{6} \mathrm{~A}\right)$-mediated initiation or internal ribosome entry site (IRES)-driven may be used to translate circRNAs into proteins. ${ }^{60}$ Several translatable circRNAs have been discovered in various tumors, including glioma. CircRNA-encoded peptides/proteins are normally fewer than 100 aa long and perform important roles EMT transition, in tumor energy metabolism, and the stabilization of the c-Myc oncoprotein. CircRNA and the peptides/proteins encoded by the circRNAs were essential in tumor cell invasion and progression. Nevertheless, peptides encoded by circRNAs were often found to be innately linked to glioma tumorigenesis, suggesting the pivotal role of circRNA in glioma malignant progressing. FBXW7-185aa is a $21-\mathrm{kDa}$ protein encoded by circ-FBXW7. By reducing the halflife of $\mathrm{c}-\mathrm{Myc}$ and antagonizing USP28-induced c-Myc stabilization, up-regulation of FBXW7-185aa blocked proliferation and cell cycle acceleration. Furthermore, in GMB samples, FBXW7-185aa and circ-FBXW7 were down-regulated, and circ-FBXW7 expression was correlated with GMB patient survival. ${ }^{61}$ CircLINC-PINT can encode a PINT87aa peptide. PINT87aa interacted with PAF1c (polymerase-associated factor 1 complex) directly and inhibited the transcriptional elongation of multiple oncogenes like c-Myc, SOX-2, and CPEB1.62 Circ-SHPRH can be translated into $17 \mathrm{kDa}$ SHPRH-146aa peptide, which is associated with glioma. In human brains, SHPRH- 146aa and circ-SHPRH are strongly expressed, but in GMB, they are down-regulated. SHPRH-146aa prevented full-length SHPRH from being degraded through the ubiquitin-proteasome, resulting in cell proliferation inhibition. ${ }^{63} \mathrm{GBM}$ progression was blocked by AKT3-174aa, which was translated by circ-AKT3 and interacted with phosphorylated PDK1 competitively. ${ }^{64}$ Bagchi found that circ 12152, a protein originating from human chromosome 9: 87482157e87570432, played a role in GMB development. ${ }^{65}$

\section{Association of circRNAs With Glioma/Glioblastoma}

Abnormal expression of circRNAs has been linked to the formation of gliomas, according to mounting data. Song and colleagues looked at RNA-seq evidence from 46 gliomas and non-cancerous brain samples and found thousands of circRNA members; 476 were expressed differentially. ${ }^{12}$ In one study, 1411 circRNAs were found to have different expressions in GBM patients. Of the 1411 circRNAs, 1205 circRNAs were down-regulated and 206 circRNAs were up-regulated; this suggests that circRNAs dysregulation is linked to the biological mechanism. ${ }^{66}$

Another research discovered that 2038 circRNAs were changed in GMB tissue compared to matched natural brain tissue. 36 circRNAs were up-regulated and 2002 circRNAs were down-regulated among the differentially expressed circRNAs. Furthermore, the development of co-expression networks indicated that changed circRNAs, which were found to be enriched in several tumor-related pathways, served as neurological disorder drivers or brain tumors. ${ }^{67}$ In both oligodendroglioma and GMB, a circRNA originating from the VCAN gene, which has been linked to gliomagenesis, was shown to be upregulated. ${ }^{12}$ In glioma, circZNF292 was reported to be down-regulated, ${ }^{68}$ whereas a circRNA originating from the $t t b k 2$ gene was found to be up-regulated. ${ }^{29}$ Finally, Barbagallo et al examined miR-671-5p expression in primary samples of GMB patient and GMB cell lines 
and discovered that it was overexpressed and associated with ciRS-7 down-regulation. ${ }^{69}$ CircRNAs' functional and identification studies indicate that they act as tumor suppressors or oncogenes that regulate downstream target genes (Table 1).

CircRNAs Act as Oncogenes in Glioma/Glioblastoma Tumorigenesis

CircZNF292 is a significant circular oncogenic RNA that affects tube forming. ${ }^{68}$ In one study, it was shown that
circZNF292 silencing inhibited cell proliferation and repressed cell cycle progression in the glioma through the Wnt/ $\beta$-catenin signaling pathway. After circZNF292 silencing, the expression levels of transcription factors STAT3, STAT5, AP-1, NF- $\kappa$ B, E2F1 and HIF-1 expression were significantly reduced, implying that circZNF292 may inhibit NF- $\kappa$ B and STAT3/5. ${ }^{68}$ In the U87MG and U251 cell lines of human glioma, suppression of circZNF292 represses glioma tube formation through the Wnt/ $\beta$ catenin signaling pathway associated genes like VEGF-A

Table 1. Expression and Functional Role of circRNAs in Glioma/Glioblastoma

\begin{tabular}{|c|c|c|c|c|}
\hline CircRNAs/Peptides & $\begin{array}{l}\text { Type of Brain } \\
\text { Tumor }\end{array}$ & $\begin{array}{l}\text { Role of Oncogenes / } \\
\text { Tumor Suppressors }\end{array}$ & $\begin{array}{l}\text { Up-regulated/Down- } \\
\text { regulated in Cancer }\end{array}$ & Function \\
\hline CircZNF292 & Glioma & Oncogene & Up-regulated & $\begin{array}{l}\text { Progression of glioma tube formation, } \\
\text { and promote glioma proliferation and } \\
\text { cell cycle progression }\end{array}$ \\
\hline Hsa_circ_0076248 & Glioma & Oncogene & Up-regulated & $\begin{array}{l}\text { Promote glioma proliferation and } \\
\text { invasion }\end{array}$ \\
\hline Circ_0034642 & Glioma & Oncogene & Up-regulated & $\begin{array}{l}\text { Promote glioma proliferation, invasion, } \\
\text { and migration, and inhibit cell apoptosis }\end{array}$ \\
\hline Circ_ 0046701 & Glioma & Oncogene & Up-regulated & Promote cell proliferation, and invasion \\
\hline Circ-0012129 & Glioma & Oncogene & Up-regulated & Promote cell proliferation, and invasion \\
\hline CircPTN & Glioma & Oncogene & Up-regulated & Promote glioma growth and stemness \\
\hline Circ_0000177 & Glioma & Oncogene & Up-regulated & $\begin{array}{l}\text { Promote glioma proliferation and } \\
\text { invasion }\end{array}$ \\
\hline CircCPA4 & Glioma & Oncogene & Up-regulated & $\begin{array}{l}\text { Regulate the proliferation and metastasis } \\
\text { of glioma }\end{array}$ \\
\hline CircSCAF11 & Glioma & Oncogene & Up-regulated & Accelerate the glioma tumorigenesis \\
\hline Circ- TTBK2 & Glioma & Oncogene & Up-regulated & $\begin{array}{l}\text { Promote proliferation and inhibited } \\
\text { apoptosis }\end{array}$ \\
\hline Hsa-circ-0014359 & Glioma & Oncogene & Up-regulated & Promote glioma progression \\
\hline Circ-U2AF1 & Glioma & Oncogene & Up-regulated & Promote glioma malignancy \\
\hline Circ-CFH & Glioma & Oncogene & Up-regulated & Promote glioma proliferation \\
\hline CircNFIX & Glioma & Oncogene & Up-regulated & Enhance glioma progression \\
\hline Circ-SHKBP1 & Glioma & Oncogene & Up-regulated & Regulate glioma angiogenesis \\
\hline Circ_002136 & Glioma & Oncogene & Up-regulated & $\begin{array}{l}\text { Promote the progression of glioma and } \\
\text { Regulate angiogenesis }\end{array}$ \\
\hline Circ-DICER1 & Glioma & Oncogene & Up-regulated & Regulate angiogenesis in glioma \\
\hline CircHIPK3 & Glioma & Oncogene & Up-regulated & $\begin{array}{l}\text { Regulate cell growth and promote glioma } \\
\text { progression }\end{array}$ \\
\hline Circ-PITX1 & Glioblastoma & Oncogene & Up-regulated & Promote the progression of glioblastoma \\
\hline CircNT5E & Glioblastoma & Oncogene & Up-regulated & $\begin{array}{l}\text { Promote proliferation, invasion, and } \\
\text { migration of cancer cells }\end{array}$ \\
\hline Circ_0029426 & Glioblastoma & Oncogene & Up-regulated & $\begin{array}{l}\text { promote cell proliferation, migration } \\
\text { and invasion, inhibited cell apoptosis of } \\
\text { Glioblastoma }\end{array}$ \\
\hline Circ-ITCH & Glioma & Tumor suppressor & Down-regulated & $\begin{array}{l}\text { Suppress cell proliferation, and promote } \\
\text { cell apoptosis }\end{array}$ \\
\hline Hsa_circ_0001649 & Glioma & Tumor suppressor & Down-regulated & $\begin{array}{l}\text { Inhibit cell growth, and facilitate } \\
\text { apoptosis }\end{array}$ \\
\hline $\begin{array}{l}\text { FBXW7-185aa encoded by } \\
\text { circ-FBXW7 }\end{array}$ & Glioma & Tumor suppressor & Down-regulated & Repress glioma \\
\hline $\begin{array}{l}\text { SHPRH-146aa encoded by } \\
\text { circRNA SHPRH }\end{array}$ & Glioma & Tumor suppressor & Down-regulated & Reduce tumorigenicity \\
\hline CircMTO1 & Glioblastoma & Tumor suppressor & Down-regulated & Inhibit glioblastoma proliferation \\
\hline Circ_0001946 & Glioblastoma & Tumor suppressor & Down-regulated & $\begin{array}{l}\text { Inhibits cell proliferation, migration, } \\
\text { invasion and induced apoptosis in } \\
\text { glioblastoma cells }\end{array}$ \\
\hline CircSMARCA5 & Glioblastoma & Tumor suppressor & Down-regulated & Anti-angiogenic function \\
\hline Circ-SHPRH & Glioblastoma & Tumor suppressor & Down-regulated & $\begin{array}{l}\text { Suppress tumor progression and } \\
\text { tumorigenesis }\end{array}$ \\
\hline $\begin{array}{l}\text { AKT3-174aa encoded by } \\
\text { circ-AKT3 }\end{array}$ & Glioblastoma & Tumor suppressor & Down-regulated & Inhibit Glioblastoma progression \\
\hline
\end{tabular}


and EGFR genes, and the VEGF-A receptor VEGFR-1/2. ${ }^{68}$ Hsa_circ_0076248 and circ_0034642 were found to facilitate glioma invasion and proliferation in miR-181a/ SIRT1 and the miR-1205/BATF3 axis, respectively. ${ }^{33,34}$ It has been discovered that hsa_circ_0076248 facilitates glioma oncogenesis. The hsa_circ_0076248 could be a sponge for miR181a, and miR181a could be a target for SIRT1 mRNA. Down-regulation of hsa_circ_0076248 or up-regulation of miR-181a could depress the invasion and proliferation of glioma in vivo and in vitro. ${ }^{33}$ It has been shown that, in tissues and cell lines of glioma circ_0034642 was up-regulated. In glioma patients, overexpression of circ_0034642 was linked to negative phenotypes. Circ_0034642 has the ability to facilitate cell invasion, proliferation, and migration, while preventing cell apoptosis. Circ_0034642 was discovered to be a miR1205 sponge, and miR-1205 has been shown to regulate BATF3 expression by targeting the $3^{\prime}$ UTR of BATF3. Circ_0034642's oncogenic function is attributed partly to its modulation of the miR-1205/BATF3 axis. The circ_0034642/miR-1205/BATF3 pathway, taken together, can play a key role in glioma. ${ }^{34} \mathrm{Li}$ and colleagues found that in glioma cell lines and tissues circ_0046701 was significantly up-regulated, and that knocking it down significantly reduced cell invasion and proliferation by upregulating miR-142-3p, which led to down-regulation of ITGB8. ${ }^{70}$ Circ-0012129 was also shown to be substantially augmented in glioma tissues, and its knockdown reduced the invasion and proliferation capabilities of SHG44 and U373 glioma lines significantly by up-regulating miR-661 expression. ${ }^{71}$ CircPTN improved Nestin, SOX9, CD133, and SOX2 expression and facilitated glioma development and stemness by sponging miR-330-5p and miR-145-5p. ${ }^{32}$ Chen et al found that in cell lines of glioma circ_0000177 was up-regulated, and that its overexpression stimulated invasion and proliferation by FZD7-induced Wnt pathway activation via miR-638 sponging. ${ }^{72}$ CircCPA4 and circSCAF11 were substantially up-regulated in glioma ${ }^{73,74}$; they accelerated glioma tumorigenesis through let7/ CPA4 and miR421/SP1/VEGFA, respectively. According to Zheng's research, circ-TTBK2 acted as a miR-217 sponge, promoting proliferation and inhibiting apoptosis in a sequence-specific manner. Furthermore, HNF1 was a direct miR-217 target and triggered Derlin-1 through ERK and PI3K/AKT signaling pathways by binding to its promoter, implying that blocking the circ-TTBK2/miR217/HNF1/Derlin-1 axis may be a possible treatment aim for human gliomas. ${ }^{29,75,76}$ MiR-153 facilitated glioma progression through the $\mathrm{PI} 3 \mathrm{~K} / \mathrm{AKT} / \mathrm{mTOR}$ signaling cascade, which was activated by circ-0014359. ${ }^{77} \mathrm{Li}$ et al found that circ-U2AF1 stimulated glioma tumorigenesis through repressing neurooncological ventral antigen 2 (NOVA2) by sponging has-miR-7-5p. ${ }^{78}$ Circ-CFH was substantially up-regulated in glioma tissue and positively associated with tumor grade, and it significantly improved glioma cell proliferative capacity by releasing AKT1 and sponging miR-149. ${ }^{79} \mathrm{Xu}$ and colleagues found that circNFIX increased development of glioma via sponging miR-34a-5p and up-regulating the target gene NOTCH1 through the Notch signaling pathway. ${ }^{80}$ Other researchers found that circ-SHKBP1 was up-regulated in glioma exposed endothelial cells (GECs) and glioma microvessels, and that it inhibited FOXP1/FOXP2 expression via targeting miR-544a/miR-379. As a result of targeting the miR-379/FOXP2/AGGF1 and miR-544a/FOXP1/AGGF1 pathways, circ-SHKBP1 modulated glioma angiogenesis. ${ }^{81}$ FUS, a member of the FET (FUS/EWS/TAF15) protein family, can regulate circ_002136 expression by binding to introns flanking the splicing site. Circ_002136 or FUS inhibition significantly reduced tube generation in U87 GECs. The FUS/circ 002136/miR-138-5p/SOX13 feedback loop has been shown to regulate angiogenesis in glioma. ${ }^{82,83}$ Another study found that MOV10 binding circ-DICER1 inhibited glioma angiogenesis in GECs by negatively regulating $\mathrm{miR}-103 \mathrm{a}-3 \mathrm{p} / \mathrm{miR}-382-5 \mathrm{p}$ on ZIC4.By triggering the PI3K/Akt pathway, ZIC4 could upregulate downstream target Hsp90 that promotes tube forming in GECs. ${ }^{84}$ CircRNAHIPK3 may sponge many miRNAs, like miR-124-3p and miR-654. ${ }^{30}$ miR-654 was indicated as a circHIPK3 target; however, miR-654 targeted IGF2BP3, circHIPK3 induced progression of glioma by the circHIPK3/miR-654/IGF2BP3 network. ${ }^{31}$ The circ-HIPK3 overexpression increased the invasive and proliferative abilities of glioma cells through sponging miR-124-3p, which increased the amount of STAT3. ${ }^{85}$ To regulate the miR-379-5p/MAP3K2 axis, Circ-PITX1 was dramatically up-regulated in GBM and facilitated GBM progression by a competing endogenous RNA. ${ }^{86}$ CircNT5E aided GMB tumorigenesis via functioning as a miR-422a sponge. ${ }^{38}$ Circ_0029426 by sponging miR-197, causing proliferation, invasion, and migration while also inhibiting GBM cell apoptosis. ${ }^{36}$

\section{CircRNAs Act as Tumor Suppressors in Glioma/ Glioblastoma}

Cir-ITCH was shown to be down-regulated in cell lines and tissues of glioma. Overexpression of cirITCH substantially stopped cell proliferation. Similarly, increased cir-ITCH expression reduced the number of established colonies while increasing the number of apoptotic cells. Cir-ITCH inhibited glioma cells' capacity to repair wounds dramatically. Glioma cells' invasive and migratory capacities were significantly reduced when cir-ITCH was up-regulated. Cir-ITCH inhibits tumor progression in glioma cells in a number of studies. ${ }^{87}$ Cir-ITCH has been shown to have an antioncogenic function by regulating the ITCH-Wnt/catenin pathway and sponging miR-214. ${ }^{87}$ In another research, hsa_circ_0001649 was shown to be reduced in glioma tissues and cell lines. Hsa_circ_0001649 that was 
down-regulated was associated to a greater tumor scale. In vivo and in vitro, hsa_circ_0001649 overexpression prevented cell development. Furthermore, up-regulated hsa_circ_0001649 aided apoptosis by regulation of the Bcl-2/caspase-3 pathway. ${ }^{88}$ CircumMTO1 expression was shown to be significantly reduced in GBM tissues. circMTO1 inhibits GBM cell proliferation through the miR-92/WWOX signaling pathway, according to Zhang et al., CircMTO1 interacts specifically with miR-92 and then functions as a miRNA sponge to increase WWOX expression. ${ }^{89}$ Circ_0001946 was found to be downregulated in GBM cells, and circ_0001946 overexpression blocked cell proliferation, invasion, triggered apoptosis, and migration in GBM cells by up-regulation of the expression of CDR1(cerebellar degeneration-related auto-antigen 1) and inhibiting miR-671-5p expression in GBM cells. The circ_0001946/miR671-5p/ CDR1 pathway has been shown to influence the progression of GBM. ${ }^{90}$ According to a recent review, circSMARCA5 serves as a sponge for the splicing factor SRSF1 (serine and arginine rich splicing factor 1), which targets and mediates VEGFA expression in GBM and hence has antiangiogenic properties. ${ }^{37}$ An FBXW7-185aa, translated from circ-FBXW7, suppressed glioma disruptive activities by antagonizing USP28-induced c-Myc stabilization. ${ }^{61}$ SHPRH-146aa, translated from the circ-SHPRH, was shown to be plentiful in typical human brains but depleted in glioma. It also decreases tumorigenicity by preserving full-length SHPRH, which acts as an E3 ligase to ubiquitinate PCNA. Both of them have a tumor suppressant function in glioma. ${ }^{61,63}$ Furthermore, AKT3$174 \mathrm{aa}$, a tumor suppressor protein encoded by circAKT3, prevented GBM development by interacting with phosphorylated PDK1. ${ }^{64}$

The role of circRNAs in Alzheimer's and Parkinson's Diseases

CircRNAs affect so many nervous system disorders, like Alzheimer's, and is caused by ciRS-7 sponging miR7. ${ }^{11}$ CDR1as/ciRS-7 is significantly miss-regulated in Alzheimer's disease patients' hippocampal CA1 area. ${ }^{92}$ CircRNAs, particularly CDR1as/ciRS-7, the bestcharacterized and most widely expressed mammalian circRNA, ${ }^{39}$ have been linked to brain degeneration and illness in several studies. CDRlas/ciRS-7 in humans contains 74 miR-7 binding sites and is aligned with the Argonaute protein in a miR-7-dependent manner. ${ }^{39,45}$ MiR-7 has been linked to neurological disorders such as Parkinson's disease. Several reports have suggested that miR-7 plays a neuroprotective function in dopaminergic neurons. MiR-7 may target the Parkinson disease-related a-synuclein protein and is active in up-regulating the mTOR pathway to defend neurons from cell death caused by the dopaminergic neurotoxic compound 1-methyl-4phenylpyridinium (MPP (+)). ${ }^{93,94}$ The circRNAs' sponge function, especially CDR1as/ciRS-7, could thus affect the progression and onset of Parkinson's disease.

\section{Future Directions and Conclusion}

Extensive studies have led to our deep understanding of the impact of complex circRNAs network in the incidence of glioma/GMB and have presented preliminary information about their role in the progression and initiation of brain tumors. Certain circRNAs are involved in some brain tumor diseases. circRNAs act as tumor suppressors or oncogenes which govern downstream target genes with notable clinical usages. Cir-ITCH, for example, is a tumor inhibitor in glioma and may be a promising predictor as a biomarker for glioma patients. Therefore, controlling the increase in cir-ITCH expression could be the future direction for developing a new therapeutic strategy. We believe that further recognition of regulatory functions of circRNAs in carcinogenic signaling pathways and greater understanding of future glioma/GBM molecular biology can help to use circRNAs as prognostic and diagnostic biomarkers and establish an application in new therapeutic targets. Identification of biomarkers with high specificity and sensitivity in the diagnosis of brain tumors, identifying people at high risk, developing targeted therapies, and evaluating the response to treatment are extensively used by many clinical experts. Although the identification of a number of circRNAs with known functions is on the rise, circRNAs research is still in its infancy, and there are still thousands of types of circRNAs with unknown functions. Most circRNAs may have a known single function or may together create a single role. It is also possible that a large fraction of the circRNAs expressed do not function specifically and are only byproducts of trimming. Therefore, knockouting circRNAs with specific genomic editing tools may help to know their performance. With advances in molecular biotechnology and full understanding of the functions of circRNAs, successful and safe circRNAs-based therapeutic strategies are hoped to be achieved in the not too distant future.

\section{Authors' contributions}

SZB provided direction in the preparation of the manuscript. SD and ADP performed primary literature search. SZB, SD and ADP wrote the first draft of manuscript. FK helped and collaborated in designing the figures. SZB discussed and revised the manuscript. ADP and SD managed the references. SZB approved the version to be published.

Conflict of Interest Disclosures

The authors declare that they have no conflict of interests.

Ethical Statement

Not applicable. 
Funding

This paper was not funded.

\section{References}

1. Ostrom QT, Cioffi G, Gittleman H, Patil N, Waite K, Kruchko C, et al. CBTRUS statistical report: primary brain and other central nervous system tumors diagnosed in the United States in 2012-2016. Neuro Oncol. 2019;21(Suppl 5):v1-v100. doi: 10.1093/neuonc/noz150.

2. Omuro A, DeAngelis LM. Glioblastoma and other malignant gliomas: a clinical review. JAMA. 2013;310(17):1842-50. doi: 10.1001/jama.2013.280319.

3. Khasraw M, Lassman AB. Advances in the treatment of malignant gliomas. Curr Oncol Rep. 2010;12(1):26-33. doi: 10.1007/s11912-009-0077-4.

4. Altieri R, Agnoletti A, Quattrucci F, Garbossa D, Calamo Specchia FM, Bozzaro M, et al. Molecular biology of gliomas: present and future challenges. Transl Med UniSa. 2014;10:29-37.

5. Rynkeviciene R, Simiene J, Strainiene E, Stankevicius V, Usinskiene J, Miseikyte Kaubriene E, et al. Non-coding RNAs in glioma. Cancers (Basel). 2018;11(1):17. doi: 10.3390/cancers 11010017.

6. Chen LL. The biogenesis and emerging roles of circular RNAs. Nat Rev Mol Cell Biol. 2016;17(4):205-11. doi: 10.1038/nrm.2015.32.

7. Patop IL, Kadener S. circRNAs in Cancer. Curr Opin Genet Dev. 2018;48:121-7. doi: https://doi.org/10.1016/j. gde.2017.11.007.

8. Shi X, Wang B, Feng X, Xu Y, Lu K, Sun M. circRNAs and exosomes: a mysterious frontier for human cancer. Mol Ther Nucleic Acids. 2020;19:384-92. doi: 10.1016/j. omtn.2019.11.023.

9. Shang Q, Yang Z, Jia R, Ge S. The novel roles of circRNAs in human cancer. Mol Cancer. 2019;18(1):6. doi: 10.1186/ s12943-018-0934-6.

10. Tang Q, Hann SS. Biological roles and mechanisms of circular RNA in human cancers. Onco Targets Ther. 2020;13:2067-92. doi: 10.2147/ott.s233672.

11. Vo JN, Cieslik M, Zhang Y, Shukla S, Xiao L, Zhang Y, et al. The landscape of circular RNA in cancer. Cell. 2019;176(4):869-81.e13. doi: 10.1016/j.cell.2018.12.021.

12. Song X, Zhang N, Han P, Moon BS, Lai RK, Wang K, et al. Circular RNA profile in gliomas revealed by identification tool UROBORUS. Nucleic Acids Res. 2016;44(9):e87. doi: 10.1093/nar/gkw075.

13. Chen N, Zhao G, Yan X, Lv Z, Yin H, Zhang S, et al. A novel FLI1 exonic circular RNA promotes metastasis in breast cancer by coordinately regulating TET1 and DNMT1. Genome Biol. 2018;19(1):218. doi: 10.1186/s13059-018$1594-\mathrm{y}$.

14. Guarnerio J, Bezzi M, Jeong JC, Paffenholz SV, Berry $\mathrm{K}$, Naldini $\mathrm{MM}$, et al. Oncogenic role of fusioncircRNAs derived from cancer-associated chromosomal translocations. Cell. 2016;165(2):289-302. doi: 10.1016/j. cell.2016.03.020.

15. Rybak-Wolf A, Stottmeister C, Glažar P, Jens $M$, Pino $\mathrm{N}$, Giusti S, et al. Circular RNAs in the mammalian brain are highly abundant, conserved, and dynamically expressed. Mol Cell. 2015;58(5):870-85. doi: 10.1016/j. molcel.2015.03.027.

16. He J, Ren M, Li H, Yang L, Wang X, Yang Q. Exosomal circular RNA as a biomarker platform for the early diagnosis of immune-mediated demyelinating disease. Front Genet. 2019;10:860. doi: 10.3389/fgene.2019.00860.

17. Gholampour S. FSI simulation of CSF hydrodynamic changes in a large population of non-communicating hydrocephalus patients during treatment process with regard to their clinical symptoms. PLoS One. 2018;13(4):e0196216. doi: 10.1371/journal.pone.0196216.

18. Gholampour S, Gholampour H. Correlation of a new hydrodynamic index with other effective indexes in Chiari I malformation patients with different associations. Sci Rep. 2020;10(1):15907. doi: 10.1038/s41598-020-72961-0.

19. Gholampour S, Fatouraee N. Boundary conditions investigation to improve computer simulation of cerebrospinal fluid dynamics in hydrocephalus patients. Commun Biol. 2021;4(1):394. doi: 10.1038/s42003-02101920-w.

20. Nakamizo S, Sasayama T, Shinohara M, Irino Y, Nishiumi $\mathrm{S}$, Nishihara M, et al. GC/MS-based metabolomic analysis of cerebrospinal fluid (CSF) from glioma patients. J Neurooncol. 2013;113(1):65-74. doi: 10.1007/s11060-0131090-x.

21. Chen W, Schuman E. Circular RNAs in brain and other tissues: a functional enigma. Trends Neurosci. 2016;39(9):597-604. doi: 10.1016/j.tins.2016.06.006.

22. You X, Vlatkovic I, Babic A, Will T, Epstein I, Tushev G, et al. Neural circular RNAs are derived from synaptic genes and regulated by development and plasticity. Nat Neurosci. 2015;18(4):603-10. doi: 10.1038/nn.3975.

23. Kristensen LS, Hansen TB, Venø MT, Kjems J. Circular RNAs in cancer: opportunities and challenges in the field. Oncogene. 2018;37(5):555-65. doi: 10.1038/onc.2017.361.

24. Li Z, Huang C, Bao C, Chen L, Lin M, Wang X, et al. Exonintron circular RNAs regulate transcription in the nucleus. Nat Struct Mol Biol. 2015;22(3):256-64. doi: 10.1038/ nsmb.2959.

25. Liu F, Zhang J, Qin L, Yang Z, Xiong J, Zhang Y, et al. Circular RNA EIF6 (Hsa_circ_0060060) sponges miR-144$3 p$ to promote the cisplatin-resistance of human thyroid carcinoma cells by autophagy regulation. Aging (Albany NY). 2018;10(12):3806-20. doi: 10.18632/aging.101674.

26. Du WW, Fang L, Yang W, Wu N, Awan FM, Yang Z, et al. Induction of tumor apoptosis through a circular RNA enhancing Foxo3 activity. Cell Death Differ. 2017;24(2):357-70. doi: 10.1038/cdd.2016.133.

27. Chen G, Wang Q, Yang Q, Li Z, Du Z, Ren M, et al. Circular RNAs hsa_circ_0032462, hsa_circ_0028173, hsa_ circ_0005909 are predicted to promote CADM1 expression by functioning as miRNAs sponge in human osteosarcoma. PLoS One. 2018;13(8):e0202896. doi: 10.1371/journal. pone.0202896.

28. Liu X, Abraham JM, Cheng Y, Wang Z, Wang Z, Zhang $\mathrm{G}$, et al. Synthetic circular RNA functions as a miR-21 sponge to suppress gastric carcinoma cell proliferation. Mol Ther Nucleic Acids. 2018;13:312-21. doi: 10.1016/j. omtn.2018.09.010. 
29. Zheng J, Liu X, Xue Y, Gong W, Ma J, Xi Z, et al. TTBK2 circular RNA promotes glioma malignancy by regulating miR-217/HNF1ß/Derlin-1 pathway. J Hematol Oncol. 2017;10(1):52. doi: 10.1186/s13045-017-0422-2.

30. Zheng Q, Bao C, Guo W, Li S, Chen J, Chen B, et al. Circular RNA profiling reveals an abundant circHIPK3 that regulates cell growth by sponging multiple miRNAs. Nat Commun. 2016;7:11215. doi: 10.1038/ncomms11215.

31. Jin P, Huang Y, Zhu P, Zou Y, Shao T, Wang O. CircRNA circHIPK3 serves as a prognostic marker to promote glioma progression by regulating miR-654/IGF2BP3 signaling. Biochem Biophys Res Commun. 2018;503(3):1570-4. doi: 10.1016/j.bbrc.2018.07.081.

32. Chen J, Chen T, Zhu Y, Li Y, Zhang Y, Wang Y, et al. circPTN sponges miR-145-5p/miR-330-5p to promote proliferation and stemness in glioma. J Exp Clin Cancer Res. 2019;38(1):398. doi: 10.1186/s13046-019-1376-8.

33. Lei B, Huang Y, Zhou Z, Zhao Y, Thapa AJ, Li W, et al. Circular RNA hsa_circ_0076248 promotes oncogenesis of glioma by sponging miR-181a to modulate SIRT1 expression. J Cell Biochem. 2019;120(4):6698-708. doi: 10.1002/jcb.27966.

34. Yang M, Li G, Fan L, Zhang G, Xu J, Zhang J. Circular RNA circ_0034642 elevates BATF3 expression and promotes cell proliferation and invasion through miR-1205 in glioma. Biochem Biophys Res Commun. 2019;508(3):980-5. doi: 10.1016/j.bbrc.2018.12.052.

35. Wang R, Zhang S, Chen X, Li N, Li J, Jia R, et al. EIF4A3induced circular RNA MMP9 (circMMP9) acts as a sponge of miR-124 and promotes glioblastoma multiforme cell tumorigenesis. Mol Cancer. 2018;17(1):166. doi: 10.1186/ s12943-018-0911-0.

36. Zhang G, Sun W, Zhu L, Feng Y, Wu L, Li T. Overexpressed circ_0029426 in glioblastoma forecasts unfavorable prognosis and promotes cell progression by sponging miR197. J Cell Biochem. 2019;120(6):10295-302. doi: 10.1002/ jcb.28313.

37. Barbagallo D, Caponnetto A, Brex D, Mirabella F, Barbagallo C, Lauretta G, et al. CircSMARCA5 regulates VEGFA mRNA splicing and angiogenesis in glioblastoma multiforme through the binding of SRSF1. Cancers (Basel). 2019;11(2):194. doi: 10.3390/cancers11020194.

38. Wang R, Zhang S, Chen X, Li N, Li J, Jia R, et al. CircNT5E acts as a sponge of miR-422a to promote glioblastoma tumorigenesis. Cancer Res. 2018;78(17):4812-25. doi: 10.1158/0008-5472.can-18-0532.

39. Hansen TB, Jensen TI, Clausen BH, Bramsen JB, Finsen B, Damgaard CK, et al. Natural RNA circles function as efficient microRNA sponges. Nature. 2013;495(7441):3848. doi: 10.1038/nature11993.

40. Hansen TB, Wiklund ED, Bramsen JB, Villadsen SB, Statham AL, Clark SJ, et al. miRNA-dependent gene silencing involving Ago2-mediated cleavage of a circular antisense RNA. EMBO J. 2011;30(21):4414-22. doi: 10.1038/emboj.2011.359.

41. Kleaveland B, Shi CY, Stefano J, Bartel DP. A network of noncoding regulatory RNAs acts in the mammalian brain. Cell. 2018;174(2):350-62.e17. doi: 10.1016/j. cell.2018.05.022.
42. Li YJ, Lei YH, Yao N, Wang CR, Hu N, Ye WC, et al. Autophagy and multidrug resistance in cancer. Chin J Cancer. 2017;36(1):52. doi: 10.1186/s40880-017-0219-2.

43. Mowers EE, Sharifi MN, Macleod KF. Autophagy in cancer metastasis. Oncogene. 2017;36(12):1619-30. doi: 10.1038/ onc.2016.333.

44. Chi G, Xu D, Zhang B, Yang F. Matrine induces apoptosis and autophagy of glioma cell line $\mathrm{U} 251$ by regulation of circRNA-104075/BCL-9. Chem Biol Interact. 2019;308:198-205. doi: 10.1016/j.cbi.2019.05.030.

45. Memczak S, Jens M, Elefsinioti A, Torti F, Krueger J, Rybak A, et al. Circular RNAs are a large class of animal RNAs with regulatory potency. Nature. 2013;495(7441):333-8. doi: 10.1038/nature11928.

46. Wang K, Long B, Liu F, Wang JX, Liu CY, Zhao B, et al. A circular RNA protects the heart from pathological hypertrophy and heart failure by targeting miR-223. Eur Heart J. 2016;37(33):2602-11. doi: 10.1093/eurheartj/ ehv713.

47. Sang Q, Liu X, Wang L, Qi L, Sun W, Wang W, et al. CircSNCA downregulation by pramipexole treatment mediates cell apoptosis and autophagy in Parkinson's disease by targeting miR-7. Aging (Albany NY). 2018;10(6):128193. doi: 10.18632/aging.101466.

48. Du WW, Zhang C, Yang W, Yong T, Awan FM, Yang BB. Identifying and characterizing circRNA-protein interaction. Theranostics. 2017;7(17):4183-91. doi: 10.7150/thno.21299.

49. Zang J, Lu D, Xu A. The interaction of circRNAs and RNA binding proteins: an important part of circRNA maintenance and function. J Neurosci Res. 2020;98(1):8797. doi: 10.1002/jnr.24356.

50. Hentze MW, Preiss T. Circular RNAs: splicing's enigma variations. EMBO J. 2013;32(7):923-5. doi: 10.1038/ emboj.2013.53.

51. Xu S, Zhou L, Ponnusamy M, Zhang L, Dong Y, Zhang Y, et al. A comprehensive review of circRNA: from purification and identification to disease marker potential. PeerJ. 2018;6:e5503. doi: 10.7717/peerj.5503.

52. Conn SJ, Pillman KA, Toubia J, Conn VM, Salmanidis $\mathrm{M}$, Phillips CA, et al. The RNA binding protein quaking regulates formation of circRNAs. Cell. 2015;160(6):112534. doi: 10.1016/j.cell.2015.02.014.

53. Khan MA, Reckman YJ, Aufiero S, van den Hoogenhof MM, van der Made I, Beqqali A, et al. RBM20 regulates circular RNA production from the titin gene. Circ Res. 2016;119(9):996-1003. doi: 10.1161/circresaha.116.309568.

54. Du WW, Yang W, Liu E, Yang Z, Dhaliwal P, Yang BB. Foxo3 circular RNA retards cell cycle progression via forming ternary complexes with p21 and CDK2. Nucleic Acids Res. 2016;44(6):2846-58. doi: 10.1093/nar/gkw027.

55. Guarnerio J, Zhang Y, Cheloni G, Panella R, Mae Katon J, Simpson $\mathrm{M}$, et al. Intragenic antagonistic roles of protein and circRNA in tumorigenesis. Cell Res. 2019;29(8):62840. doi: 10.1038/s41422-019-0192-1.

56. Li X, Wang J, Zhang C, Lin C, Zhang J, Zhang W, et al. Circular RNA circITGA7 inhibits colorectal cancer growth and metastasis by modulating the Ras pathway and upregulating transcription of its host gene ITGA7. J Pathol. 
2018;246(2):166-79. doi: 10.1002/path.5125.

57. Granados-Riveron JT, Aquino-Jarquin G. The complexity of the translation ability of circRNAs. Biochim Biophys Acta. 2016;1859(10):1245-51. doi: 10.1016/j. bbagrm.2016.07.009.

58. Abe N, Matsumoto K, Nishihara M, Nakano Y, Shibata A, Maruyama $\mathrm{H}$, et al. Rolling circle translation of circular RNA in living human cells. Sci Rep. 2015;5:16435. doi: 10.1038/srep16435.

59. Wilusz JE. Circular RNAs: unexpected outputs of many protein-coding genes. RNA Biol. 2017;14(8):1007-17. doi: 10.1080/15476286.2016.1227905.

60. Wu P, Mo Y, Peng M, Tang T, Zhong Y, Deng X, et al. Emerging role of tumor-related functional peptides encoded by lncRNA and circRNA. Mol Cancer. 2020;19(1):22. doi: 10.1186/s12943-020-1147-3.

61. Yang Y, Gao X, Zhang M, Yan S, Sun C, Xiao F, et al. Novel role of FBXW7 circular RNA in repressing glioma tumorigenesis. J Natl Cancer Inst. 2018;110(3):304-15. doi: 10.1093/jnci/djx166.

62. Zhang M, Zhao K, Xu X, Yang Y, Yan S, Wei P, et al. A peptide encoded by circular form of LINC-PINT suppresses oncogenic transcriptional elongation in glioblastoma. Nat Commun. 2018;9(1):4475. doi: 10.1038/s41467-01806862-2.

63. Zhang M, Huang N, Yang X, Luo J, Yan S, Xiao F, et al. A novel protein encoded by the circular form of the SHPRH gene suppresses glioma tumorigenesis. Oncogene. 2018;37(13):1805-14. doi: 10.1038/s41388-017-0019-9.

64. Xia X, Li X, Li F, Wu X, Zhang M, Zhou H, et al. A novel tumor suppressor protein encoded by circular AKT3 RNA inhibits glioblastoma tumorigenicity by competing with active phosphoinositide-dependent Kinase-1. Mol Cancer. 2019;18(1):131. doi: 10.1186/s12943-019-1056-5.

65. Bagchi A. Different roles of circular RNAs with protein coding potentials. Biochem Biophys Res Commun. 2018;500(4):907-9. doi: 10.1016/j.bbrc.2018.04.190.

66. Zhu J, Ye J, Zhang L, Xia L, Hu H, Jiang H, et al. Differential expression of circular RNAs in glioblastoma multiforme and its correlation with prognosis. Transl Oncol. 2017;10(2):271-9. doi: 10.1016/j.tranon.2016.12.006.

67. Yuan Y, Jiaoming L, Xiang W, Yanhui L, Shu J, Maling G, et al. Analyzing the interactions of mRNAs, miRNAs, lncRNAs and circRNAs to predict competing endogenous RNA networks in glioblastoma. J Neurooncol. 2018;137(3):493502. doi: 10.1007/s11060-018-2757-0.

68. Yang P, Qiu Z, Jiang Y, Dong L, Yang W, Gu C, et al. Silencing of cZNF292 circular RNA suppresses human glioma tube formation via the $\mathrm{Wnt} / \beta$-catenin signaling pathway. Oncotarget. 2016;7(39):63449-55. doi: 10.18632/ oncotarget.11523.

69. Barbagallo D, Condorelli A, Ragusa M, Salito L, Sammito M, Banelli B, et al. Dysregulated miR-671-5p / CDR1AS / CDR1 / VSNL1 axis is involved in glioblastoma multiforme. Oncotarget. 2016;7(4):4746-59. doi: 10.18632/ oncotarget.6621.

70. Li G, Yang H, Han K, Zhu D, Lun P, Zhao Y. A novel circular RNA, hsa_circ_0046701, promotes carcinogenesis by increasing the expression of miR-142-3p target ITGB8 in glioma. Biochem Biophys Res Commun. 2018;498(1):25461. doi: 10.1016/j.bbrc.2018.01.076.

71. Xie G. Circular RNA hsa-circ-0012129 promotes cell proliferation and invasion in 30 cases of human glioma and human glioma cell lines U373, A172, and SHG44, by targeting microRNA-661 (miR-661). Med Sci Monit. 2018;24:2497-507. doi: 10.12659/msm.909229.

72. Chen Z, Duan X. hsa_circ_0000177-miR-638-FZD7-Wnt signaling cascade contributes to the malignant behaviors in glioma. DNA Cell Biol. 2018;37(9):791-7. doi: 10.1089/ dna.2018.4294.

73. Meng Q, Li S, Liu Y, Zhang S, Jin J, Zhang Y, et al. Circular RNA circSCAF11 accelerates the glioma tumorigenesis through the miR-421/SP1/VEGFA axis. Mol Ther Nucleic Acids. 2019;17:669-77. doi: 10.1016/j.omtn.2019.06.022.

74. Peng H, Qin C, Zhang C, Su J, Xiao Q, Xiao Y, et al. circCPA4 acts as a prognostic factor and regulates the proliferation and metastasis of glioma. J Cell Mol Med. 2019;23(10):6658-65. doi: 10.1111/jcmm.14541.

75. Dong QZ, Wang Y, Tang ZP, Fu L, Li QC, Wang ED, et al. Derlin-1 is overexpressed in non-small cell lung cancer and promotes cancer cell invasion via EGFR-ERK-mediated up-regulation of MMP-2 and MMP-9. Am J Pathol. 2013;182(3):954-64. doi: 10.1016/j.ajpath.2012.11.019.

76. Tan X, He X, Jiang Z, Wang X, Ma L, Liu L, et al. Derlin-1 is overexpressed in human colon cancer and promotes cancer cell proliferation. Mol Cell Biochem. 2015;408(1-2):205-13. doi: 10.1007/s11010-015-2496-x.

77. Shi F, Shi Z, Zhao Y, Tian J. CircRNA hsa-circ-0014359 promotes glioma progression by regulating miR-153/ PI3K signaling. Biochem Biophys Res Commun. 2019;510(4):614-20. doi: 10.1016/j.bbrc.2019.02.019.

78. Li G, Huang M, Cai Y, Yang Y, Sun X, Ke Y. Circ-U2AF1 promotes human glioma via derepressing neurooncological ventral antigen 2 by sponging hsa-miR-7-5p. J Cell Physiol. 2019;234(6):9144-55. doi: 10.1002/jcp.27591.

79. Bian A, Wang Y, Liu J, Wang X, Liu D, Jiang J, et al. Circular RNA complement factor $\mathrm{H}(\mathrm{CFH})$ promotes glioma progression by sponging miR-149 and regulating AKT1. Med Sci Monit. 2018;24:5704-12. doi: 10.12659/ msm.910180.

80. Xu H, Zhang Y, Qi L, Ding L, Jiang H, Yu H. NFIX circular RNA promotes glioma progression by regulating miR$34 a-5 p$ via notch signaling pathway. Front Mol Neurosci. 2018;11:225. doi: 10.3389/fnmol.2018.00225.

81. He Q, Zhao L, Liu Y, Liu X, Zheng J, Yu H, et al. circSHKBP1 regulates the angiogenesis of U87 glioma-exposed endothelial cells through miR-544a/FOXP1 and miR-379/ FOXP2 pathways. Mol Ther Nucleic Acids. 2018;10:331-48. doi: 10.1016/j.omtn.2017.12.014.

82. Verheijen BM, Pasterkamp RJ. Commentary: FUS affects circular RNA expression in murine embryonic stem cellderived motor neurons. Front Mol Neurosci. 2017;10:412. doi: 10.3389/fnmol.2017.00412.

83. He Z, Ruan X, Liu X, Zheng J, Liu Y, Liu L, et al. FUS/ circ_002136/miR-138-5p/SOX13 feedback loop regulates angiogenesis in glioma. J Exp Clin Cancer Res. 2019;38(1):65. doi: 10.1186/s13046-019-1065-7.

84. He Q, Zhao L, Liu X, Zheng J, Liu Y, Liu L, et al. MOV10 
binding circ-DICER1 regulates the angiogenesis of glioma via miR-103a-3p/miR-382-5p mediated ZIC4 expression change. J Exp Clin Cancer Res. 2019;38(1):9. doi: 10.1186/ s13046-018-0990-1.

85. Hu D, Zhang Y. Circular RNA HIPK3 promotes glioma progression by binding to miR-124-3p. Gene. 2019;690:819. doi: 10.1016/j.gene.2018.11.073.

86. Lv X, Wang M, Qiang J, Guo S. Circular RNA circ-PITX1 promotes the progression of glioblastoma by acting as a competing endogenous RNA to regulate miR-379-5p/ MAP3K2 axis. Eur J Pharmacol. 2019;863:172643. doi: 10.1016/j.ejphar.2019.172643.

87. Li F, Ma K, Sun M, Shi S. Identification of the tumorsuppressive function of circular RNA ITCH in glioma cells through sponging miR-214 and promoting linear ITCH expression. Am J Transl Res. 2018;10(5):1373-86.

88. Wang Y, Sui X, Zhao H, Cong L, Li Y, Xin T, et al. Decreased circular RNA hsa_circ_0001649 predicts unfavorable prognosis in glioma and exerts oncogenic properties in vitro and in vivo. Gene. 2018;676:117-22. doi: 10.1016/j. gene.2018.07.037.

89. Zhang X, Zhong B, Zhang W, Wu J, Wang Y. Circular RNA
CircMTO1 inhibits proliferation of glioblastoma cells via miR-92/WWOX signaling pathway. Med Sci Monit. 2019;25:6454-61. doi: 10.12659/msm.918676.

90. Li X, Diao H. Circular RNA circ_0001946 acts as a competing endogenous RNA to inhibit glioblastoma progression by modulating miR-671-5p and CDR1. J Cell Physiol. 2019;234(8):13807-19. doi: 10.1002/jcp.28061.

91. Akhter R. Circular RNA and Alzheimer's disease. Adv Exp Med Biol. 2018;1087:239-43. doi: 10.1007/978-981-131426-1_19.

92. Lukiw WJ. Circular RNA (circRNA) in Alzheimer's disease (AD). Front Genet. 2013;4:307. doi: 10.3389/ fgene.2013.00307.

93. Choi DC, Chae YJ, Kabaria S, Chaudhuri AD, Jain MR, $\mathrm{Li} \mathrm{H}$, et al. MicroRNA-7 protects against 1-methyl4-phenylpyridinium-induced cell death by targeting RelA. J Neurosci. 2014;34(38):12725-37. doi: 10.1523/ jneurosci.0985-14.2014.

94. Fragkouli A, Doxakis E. miR-7 and miR-153 protect neurons against $\mathrm{MPP}(+)$-induced cell death via upregulation of mTOR pathway. Front Cell Neurosci. 2014;8:182. doi: $10.3389 /$ fncel.2014.00182 